\title{
Adaptive Exponential Synchronization of Coupled Complex Networks on General Graphs
}

\author{
Song Liu, Xianfeng Zhou, Wei Jiang, and Yizheng Fan \\ School of Mathematical Sciences, Anhui University, Hefei 230601, China \\ Correspondence should be addressed to Song Liu; liusong@ahu.edu.cn
}

Received 4 December 2012; Accepted 23 March 2013

Academic Editor: Allan Peterson

Copyright (c) 2013 Song Liu et al. This is an open access article distributed under the Creative Commons Attribution License, which permits unrestricted use, distribution, and reproduction in any medium, provided the original work is properly cited.

\begin{abstract}
We investigate the synchronization in complex dynamical networks, where the coupling configuration corresponds to a weighted graph. An adaptive synchronization method on general coupling configuration graphs is given. The networks may synchronize at an arbitrarily given exponential rate by enhancing the updated law of the variable coupling strength and achieve synchronization more quickly by adding edges to original graphs. Finally, numerical simulations are provided to illustrate the effectiveness of our theoretical results.
\end{abstract}

\section{Introduction}

In general, complex networks consist of a large number of nodes and links among them, in which a node is a fundamental cell with specific activity, hence, complex networks and graphs closely contact each other. The dynamics on complex networks is one on graphs, though the graphs may have different characteristic, for example, classical random graph model [1], small-world model [2-4], scale-free model [5], or others that are closely related to natural structure.

Synchronization of coupled complex dynamical networks is a universal phenomenon in various fields of science and society. All kinds of synchronization, including adaptive synchronization, global synchronization, antisynchronization, phase synchronization, projection synchronization, and generalized synchronization have been studied [6-14], in particular on the synchronization of an array of linearly coupled identical systems [15-18]. Researches [15, 19] imply that the structural properties of a network must have inevitable effect on the ability and speed of synchronization, but such work still does not see more. In addition, as pointed in [18], the coupling strength may be self-adaptive due to the spontaneousness of updated law, not be calculated numerically in many other works.

In the paper [18], an adaptive synchronization method is introduced, and the networks can synchronize by enhancing the coupling strength automatically under a simple updated law. However, their work is limited to tree-like networks and cannot be applied to general networks. In fact, a tree is a graph without cycles; in this paper, we try to extend the work in [18] to general networks or graphs. We find that their method is in fact effective to general graphs by a rigorous proof, and the networks can also achieve synchronization at an arbitrarily given exponential rate by increasing coupling strength. Based on the knowledge of spectral graph theory, networks can synchronize more quickly by increasing the algebraic connectivity of the graphs, which can be realized by adding edges to original graphs. Finally, numerical simulations are provided to illustrate the effectiveness of theoretical results.

\section{Preliminaries}

In this section, we now introduce some notations and preliminaries.

Consider the complex dynamical network consisting of $N$ linearly and diffusively coupled identical nodes with full diagonal coupling, and each node is an $n$-dimensional dynamical oscillator which may be chaotic. The state equations of the network are

$$
\dot{x}_{i}=f\left(x_{i}\right)+c \sum_{j=1}^{N} a_{i j} \Gamma x_{j}, \quad i=1,2, \ldots, N,
$$

where $x_{i}=\left(x_{i 1}, x_{i 2}, \ldots, x_{i n}\right)^{T} \in \mathbb{R}^{n}$ is a state vector of node $i$, $f\left(x_{i}\right)=\left(f_{1}\left(x_{i}\right), f_{2}\left(x_{i}\right), \ldots, f_{n}\left(x_{i}\right)\right)^{T}: \mathbb{R}^{n} \rightarrow \mathbb{R}^{n}$ is a given 
nonlinear vector valued function describing the dynamics of the nodes, $c>0$ represents coupling strength, and the inner coupling link matrix is a diagonal matrix $\Gamma=$ $\operatorname{diag}\left\{r_{1}, r_{2}, \ldots, r_{n}\right\}$ with $r_{i}>0$. The coupling configuration matrix $A=\left(a_{i j}\right)_{N \times N}$ is a zero row sums matrix with nonnegative off-diagonal entries, representing the topological structure of the network.

There is a weighted graph corresponding to the coupling configuration matrix $A$, called the coupling configuration graph, defined as a graph $G$ on vertices $1,2, \ldots, N$ which contains an edge $i j(i \neq j)$ with weight $a_{i j}$ if and only if $a_{i j}>0$. Giving an arbitrary orientation of the edges of $G$, so that each edge has a head and a tail, and a labeling of edges as $e_{1}, e_{2}, \ldots, e_{E}$, where $E$ denotes the number of edges of $G$. We obtain an edge-vertex incidence matrix of $G$, denoted as $M:=$ $M(A)=\left(m_{i j}\right)_{E \times N}$, which is defined as $m_{i j}=\sqrt{a_{i j}}$ (resp., $m_{i j}=-\sqrt{a_{i j}}$ ) if the the edge $e_{i}$ has the vertex $j$ as a head (resp., a tail), and $m_{i j}=0$ otherwise. The Laplacian matrix of $G$ is defined as $L=M^{T} M=D-B$, where $D$ is a diagonal matrix, and its $i$ th diagonal entry of which is exactly the degree of the vertex $i$, that is, $d_{i}=\sum_{j \in N(i)} a_{i j}$, where $N(i)$ is the set of neighbors of $i$ in the graph $G$ (or the vertices joining $i$ by edges), and $B=\left(b_{i j}\right)$ is a weighted adjacency matrix of $G$ such that $b_{i j}=a_{i j}$ if $i j$ is an edge of $G$ and $b_{i j}=0$ otherwise. One can find that $L=-A$ and is symmetric and positive semidefinite, so its eigenvalues can be arranged as

$$
0=\lambda_{0} \leq \lambda_{1} \leq \lambda_{2} \leq \cdots \leq \lambda_{N-1}
$$

where $\lambda_{0}=0$ as $L$ has zero row sums, and $\lambda_{1}>0$ if and only if $G$ is connected and is called the algebraic connectivity of $G$ by Fiedler [20] in the case of $G$ is simple (i.e., all edges have weight 1 ). If $G$ is connected, the corresponding eigenvector of the eigenvalue 0 is an all one vector (up to a scalar multiple), denoted by $\mathbf{1}$. One can refer to Chung [21] and Merris [22] for the details of Laplacian matrices of graphs.

If we denote $x=\left(x_{1}^{T}, x_{2}^{T}, \ldots, x_{N}^{T}\right)^{T}, F(x)=\left(f^{T}\left(x_{1}\right)\right.$, $\left.f^{T}\left(x_{2}\right), \ldots, f^{T}\left(x_{N}\right)\right)^{T}$ and substitute $-L$ for $A,(1)$ is transformed as

$$
\dot{x}=F(x)-c L \otimes \Gamma x .
$$

Taking the transformation $y=x e^{a t}(a>0),(3)$ is written as

$$
\dot{y}=e^{a t} F\left(e^{-a t} y\right)-\left(c L \otimes \Gamma-a I_{N n}\right) y,
$$

where $I_{N n}=I_{N} \otimes I_{n}$, and $I_{m}$ denotes an identity matrix of order $m$.

In this paper, we adopt the $l_{2}$-norm for vectors and the induced spectral norm for matrices. We always suppose that the function $F$ in (3) is Lipschitz continuous, or equivalently $f$ in (1) is Lipschitz continuous, that is, there exists a constant $l>0$ such that for any $x, y \in R^{n}$,

$$
\|f(x)-f(y)\| \leq l \cdot\|x-y\| .
$$

Denote by $\mathbf{M}=M \otimes I_{n}$, one can obtain the following lemma.
Lemma 1 (see [15]). Suppose that the coupling configuration graph corresponding to $A=-L$ is connected. Then, the dynamical networks (4) achieve synchronization if and only if $\lim _{t \rightarrow \infty}\|\mathbf{M} y\|=0$.

\section{Main Results}

If the network (4) achieves synchronization, surely the network (3) or (1) achieves synchronization with exponential rate $a$. So, we directly discuss (4), the main result of this paper is stated as follows.

Theorem 2. Suppose that $F$ is Lipschitz continuous, and the coupling configuration graph corresponding to $A=-L$ is connected. Then, the network (4) achieves synchronization, and in particular the network (1) achieves exponential synchronization with rate $a$, when the coupling strength $c$ is adapted duly according to the following updated law:

$$
\dot{c}=k y^{T}(t)\left(L^{2} \otimes \Gamma\right) y(t)
$$

where $k>0$ is an arbitrary constant.

Proof. Construct a Lyapunov function

$$
V(t)=\frac{1}{2}\|\mathbf{M} y(t)\|^{2}+\frac{1}{2 k}(c-h)^{2},
$$

where $h$ is a sufficiently large constant.

Noting that $\mathbf{M}^{T} \mathbf{M}=L \otimes I_{n}$, and substituting (6) for $\dot{c}$, we get the derivative of $V(t)$ along the trajectories of (4) as follows:

$$
\begin{aligned}
\dot{V}= & (\mathbf{M} y)^{T}(\mathbf{M} \dot{y})+\frac{1}{k}(c-h) \dot{c} \\
= & (\mathbf{M} y)^{T} \mathbf{M}\left[e^{a t} F\left(e^{-a t} y\right)-\left(c L \otimes \Gamma-a I_{N n}\right) y\right] \\
& +(c-h) y^{T}\left(L^{2} \otimes \Gamma\right) y \\
= & e^{a t}(\mathbf{M} y)^{T} \mathbf{M} F\left(e^{-a t} y\right)-h y^{T}\left(L^{2} \otimes \Gamma\right) y+a y^{T}\left(L \otimes I_{n}\right) y \\
& -c y^{T} \mathbf{M}^{T} \mathbf{M}(L \otimes \Gamma) y+c y^{T}\left(L^{2} \otimes \Gamma\right) y .
\end{aligned}
$$

Noting that

$$
\mathbf{M}^{T} \mathbf{M}(L \otimes \Gamma)=\left(L \otimes I_{n}\right)(L \otimes \Gamma)=L^{2} \otimes \Gamma,
$$

we have

$$
\dot{V}=e^{a t}(\mathbf{M} y)^{T} \mathbf{M} F\left(e^{-a t} y\right)-h y^{T}\left(L^{2} \otimes \Gamma\right) y+a y^{T}\left(L \otimes I_{n}\right) y .
$$

Let $G$ be the coupling configuration graph corresponding to $A=-L$. Let $\vec{G}$ be the directed graph after an arbitrary orientation of the edges of $G$, and let $\mathscr{E}(\vec{G})$ be the edge set of $\vec{G}$. An oriented edge in $\mathscr{E}(\vec{G})$ is denoted by $e=i \rightarrow j$, where $j$ is the head of $e$ and $i$ is the tail of $e$. 
Observe that

$$
\begin{aligned}
& (\mathbf{M} y)^{T} \mathbf{M} F\left(e^{-a t} y\right) \\
& =\sum_{e=i \rightarrow j \in \mathscr{E}(\vec{G})} m_{i j}\left(y_{j}-y_{i}\right)^{T} \cdot m_{i j}\left[f\left(e^{-a t} y_{j}\right)-f\left(e^{-a t} y_{i}\right)\right] \\
& \leq e^{-a t} \cdot l \cdot \sum_{e=i \rightarrow j \in \mathscr{E}(\vec{G})} m_{i j}^{2}\left\|y_{j}-y_{i}\right\|^{2} \\
& =l e^{-a t}(\mathbf{M} y)^{T}(\mathbf{M} y)=l e^{-a t} y^{T}\left(L \otimes I_{n}\right) y .
\end{aligned}
$$

Hence,

$$
\begin{aligned}
\dot{V} & \leq(l+a) y^{T}\left(L \otimes I_{n}\right) y-h y^{T}\left(L^{2} \otimes \Gamma\right) y \\
& \leq(l+a) y^{T}\left(L \otimes I_{n}\right) y-h r y^{T}\left(L^{2} \otimes I_{n}\right) y,
\end{aligned}
$$

where $r=\min _{1 \leq i \leq n} r_{i}>0$.

Note that $L$ is a real and symmetric matrix, so we may let $z_{0}:=(1 / \sqrt{n}) \mathbf{1}, z_{1}, \ldots, z_{N-1}$ as unit orthogonal eigenvectors corresponding to eigenvalues $\lambda_{0}=0, \lambda_{1}, \ldots, \lambda_{N-1}$ of the matrix $L$, respectively. Let $\bar{z}_{0}, \bar{z}_{1}, \ldots, \bar{z}_{n-1}$ be an arbitrary orthogonal base of $\mathbb{R}^{n}$. Thus, $z_{i} \otimes \bar{z}_{j}, i=0,1, \ldots, N-1$, $j=0,1, \ldots, n-1$, form an orthogonal base of $\mathbb{R}^{N n}$, which are also the unit orthogonal eigenvectors of $L \otimes I_{n}$ and $L^{2} \otimes I_{n}$. As the associated graph of $L$ or $A$ is connected, $\lambda_{1}>0$, we now write

$$
y(t)=\sum_{i, j} \alpha_{i j}(t) z_{i} \otimes \bar{z}_{j}
$$

Then, we obtain

$$
\begin{aligned}
\dot{V} & \leq(l+a) \sum_{i \geq 0, j} \lambda_{i} \alpha_{i j}(t)^{2}-h r \sum_{i \geq 0, j} \lambda_{i}^{2} \alpha_{i j}(t)^{2} \\
& =(l+a) \sum_{i \geq 1, j} \lambda_{i} \alpha_{i j}(t)^{2}-h r \sum_{i \geq 1, j} \lambda_{i}^{2} \alpha_{i j}(t)^{2} \\
& \leq(l+a) \sum_{i \geq 1, j} \lambda_{i} \alpha_{i j}(t)^{2}-h r \sum_{i \geq 1, j} \lambda_{i} \lambda_{1} \alpha_{i j}(t)^{2} \\
& =\left(l+a-h r \lambda_{1}\right) \sum_{i \geq 1, j} \lambda_{i} \alpha_{i j}(t)^{2} \\
& =\left(l+a-h r \lambda_{1}\right) y^{T}\left(L \otimes I_{n}\right) y \\
& =\left(l+a-h r \lambda_{1}\right)(\mathbf{M} y)^{T}(\mathbf{M} y) .
\end{aligned}
$$

Hence, we can choose a sufficiently large $h$ such that $\beta:=l+$ $a-h r \lambda_{1}<0$. Thus,

$$
\dot{V} \leq \beta(\mathbf{M} y(t))^{T}(\mathbf{M} y(t)) .
$$

It is obvious that $\dot{V}=0$ if and only if $\mathbf{M} y=0$. In addition, if $\mathbf{M} y=0$, then $y=\mathbf{1} \otimes y_{1}$, and $\dot{V}=0$ by (10). Therefore, the set $E=\{y \mid \mathbf{M} y=0\}$ is the largest invariant set contained in $\dot{V}=0$ for the system (4). Then, we obtain $\lim _{t \rightarrow \infty}\|\mathbf{M} y\|=0$ based on the LaSalle invariant principle of differential equations. Hence, by Lemma 1, the complex dynamical network (4) is synchronized under the updated

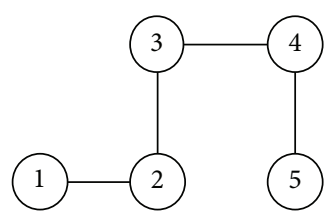

(a)

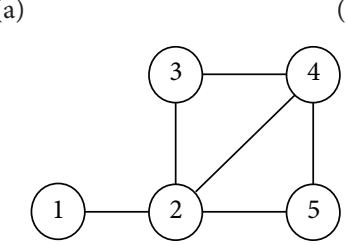

(c)

FIGURE 1: Three small networks.

law (6) of coupling strength $c$. In particular, the complex dynamical network (1) is exponentially synchronized with rate $a$.

Remark 3. In [18], the updated law of coupling strength $c$ is given as follows:

$$
\dot{c}=K x(t)^{T} \mathbf{M}^{T} \mathbf{M M}^{T} \mathbf{M} \Gamma x(t)=K x(t)^{T} L^{2} \otimes \Gamma x(t),
$$

where $\boldsymbol{\Gamma}=I_{N} \otimes \Gamma$; the synchronization speed of complex dynamical network (1) or (3) can be controlled by the constant $K$ in (16). Here, we take $k=K e^{2 a t}$; the synchronization is realized with exponential rate $a$. So, we make a further illustration of the above statement in [18] from (6) and Theorem 2.

Remark 4. From (15) in the proof of Theorem 2 , if $\beta$ is taken smaller, $V(t)$ will decrease quickly, and then the synchronization of the network will be attained quickly. This will be done if the Laplacian matrix of the coupling configuration graph has a larger eigenvalue $\lambda_{1}$. Based on the knowledge of spectral graph theory (or see [23]), $\lambda_{1}$ is not decreased by adding edges to original graph. Therefore, we can add edges to the coupling configuration graph such that the complex network achieves synchronization more quickly.

Remark 5. As the coupling configuration graph is connected and Lemma 1 holds, the dynamical network (4) achieves synchronization. If the graph is disconnected, the network only can synchronize in each connected component of the graph but not in the whole graph.

\section{Numerical Simulations}

In this section, we present several numerical examples to show the theoretical results. In particular, we consider complex dynamical networks with the Lorenz system as each node

$$
\left[\begin{array}{l}
\dot{x}_{i 1} \\
\dot{x}_{i 2} \\
\dot{x}_{i 3}
\end{array}\right]=\left[\begin{array}{l}
\alpha\left(x_{i 2}-x_{i 1}\right) \\
\gamma x_{i 1}-x_{i 1} x_{i 3}-x_{i 2} \\
x_{i 1} x_{i 2}-\beta x_{i 3}
\end{array}\right], \quad i=1,2,3,4,5,
$$

which is chaotic when $\alpha=10, \beta=8 / 3, \gamma=28$. Three small networks with five nodes are shown in Figure 1, where the 


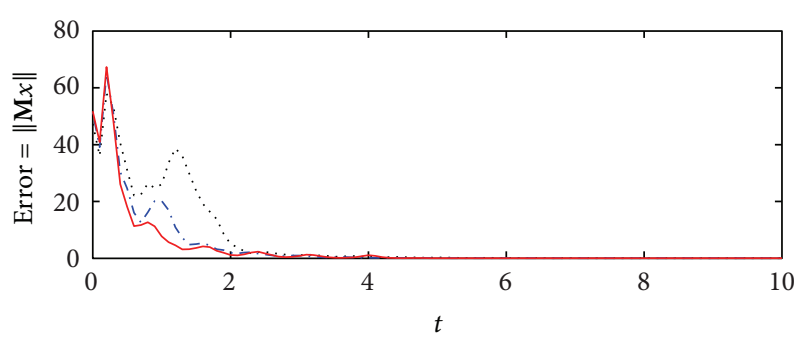

…. Figure $1(\mathrm{a})$

-. - Figure 1(b)

- Figure 1(c)

(a)

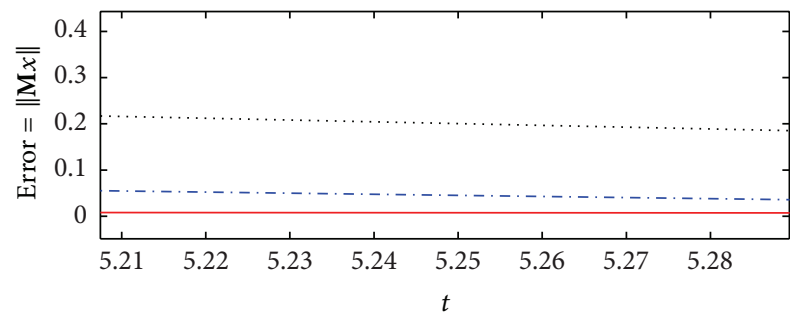

..... Figure $1(\mathrm{a})$

...- Figure 1(b)

— Figure 1(c)

(b)

FIGURE 2: Synchronization errors $\|\mathbf{M} x\|$ of three networks in Figure 1.

weight of every edge in each network is 1 . Choose the inner coupling link matrix to be $\Gamma=\operatorname{diag}\{1,2,3\}$.

The corresponding coupling configuration matrices are listed in the same order as follows:

$$
\left[\begin{array}{ccccc}
-1 & 1 & 0 & 0 & 0 \\
1 & -2 & 1 & 0 & 0 \\
0 & 1 & -2 & 1 & 0 \\
0 & 0 & 1 & -2 & 1 \\
0 & 0 & 0 & 1 & -1
\end{array}\right], \quad\left[\begin{array}{ccccc}
-1 & 1 & 0 & 0 & 0 \\
1 & -3 & 1 & 0 & 1 \\
0 & 1 & -2 & 1 & 0 \\
0 & 0 & 1 & -2 & 1 \\
0 & 1 & 0 & 1 & -2
\end{array}\right],
$$

$$
\left[\begin{array}{ccccc}
-1 & 1 & 0 & 0 & 0 \\
1 & -4 & 1 & 1 & 1 \\
0 & 1 & -2 & 1 & 0 \\
0 & 1 & 1 & -3 & 1 \\
0 & 1 & 0 & 1 & -2
\end{array}\right]
$$

According to Theorem 2, the updated law of coupling strength $c$ is chosen as $\dot{c}=K x^{T}(t)\left(L^{2} \otimes \Gamma\right) x(t)$ (i.e., $k=$ $K, a=0$ in (6)). Here $K=0.0001$, the initial coupling strength $c=0$, and the initial value of $x$ is taken as $[-8,2$, $35,1,-9,-3,-4,5,1,2,-4,1,-7,8,4]^{T}$. The synchronization errors \| $\mathbf{M} x \|$ of three networks are shown in Figure 2, respectively, where the figure listed below is a local magnification of the above figure, and Figure 2 also shows that the method in [18] is effective to general networks. Numerical simulations show that synchronization can be reached more quickly by increasing the algebraic connectivity of the graph, which can be realized by adding edges to original graph.

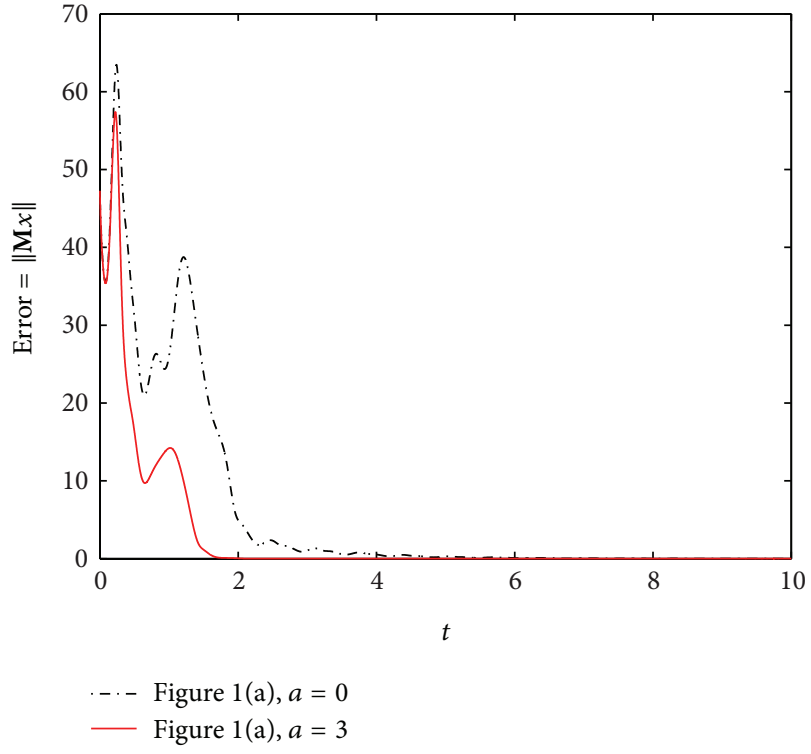

FIGURE 3: Exponential Synchronization errors $\|\mathbf{M} x\|$ of the network in Figure 1(a).

Figure 3 shows that adaptive exponential synchronization is achieved for complex dynamical network in Figure 1(a), where $k=0.0001, a=0$, and $a=3$ in (6). The same results also hold for the networks in Figures 1(b) and 1(c).

\section{Conclusions}

In this paper, an adaptive synchronization method on general networks or graphs is obtained. According to our method, networks may synchronize at an arbitrarily given exponential rate by increasing coupling strength and synchronize more quickly by increasing the algebraic connectivity of the corresponding graphs, which can be achieved by adding edges to original graphs. The obtained results also extend the work in [18] as a tree is a graph without cycles. Finally, numerical simulations are provided to illustrate the effectiveness of our theoretical results.

\section{Acknowledgments}

This research is supported by National Natural Science Foundation of China (11071001, 11071002), Major Program of Educational Commission of Anhui Province of China (KJ2010ZD02), Program of Natural Science of Colleges of Anhui Province (KJ2013A032, KJ2011A020, KJ2012B040), Starting Research Fund of Anhui University (023033190181), Young Scientist Fund of Anhui University (023033050055), and 211 Project of Anhui University (KJTD002B).

\section{References}

[1] P. Erdős and A. Rényi, "On random graphs. I," Publicationes Mathematicae Debrecen, vol. 6, pp. 290-297, 1959.

[2] D. J. Watts and S. H. Strogatz, "Collective dynamics of "smallworld” networks," Nature, vol. 393, pp. 440-442, 1998. 
[3] M. E. J. Newman and D. J. Watts, "Scaling and percolation in the small-world network model," Physical Review E, vol. 60, no. 6, pp. 7332-7342, 1999.

[4] M. Barahnoa and L. M. Pecora, "Synchronization in smallworld systems," Physical Review Letters, vol. 89, no. 5, Article ID 054101, 4 pages, 2002.

[5] A. L. Barabási, H. Jeong, and R. Albert, "Scale-free characteristics of random networks: the topology of the world wide web," Physica A, vol. 281, no. 1-4, pp. 69-77, 2000.

[6] Y. Chen, X. F. Wu, and Z. F. Gui, "Global synchronization criteria for a class of third-order non-autonomous chaotic systems via linear state error feedback control," Applied Mathematical Modelling, vol. 34, no. 12, pp. 4161-4170, 2010.

[7] G. Fu and Z. Li, "Robust adaptive anti-synchronization of two different hyperchaotic systems with external uncertainties," Communications in Nonlinear Science and Numerical Simulation, vol. 16, no. 1, pp. 395-401, 2011.

[8] S. Liu, X. Li, W. Jiang, and Y.-Z. Fan, "Adaptive synchronization in complex dynamical networks with coupling delays for general graphs," Applied Mathematics and Computation, vol. 219, no. 1, pp. 83-87, 2012.

[9] H. Taghvafard and G. H. Erjaee, "Phase and anti-phase synchronization of fractional order chaotic systems via active control," Communications in Nonlinear Science and Numerical Simulation, vol. 16, no. 10, pp. 4079-4088, 2011.

[10] D. Ghosh, S. Banerjee, and A. R. Chowdhury, "Generalized and projective synchronization in modulated time-delayed systems," Physics Letters A, vol. 374, pp. 2143-2149, 2010.

[11] M. Sun, C. Zeng, and L. Tian, "Linear generalized synchronization between two complex networks," Communications in Nonlinear Science and Numerical Simulation, vol. 15, no. 8, pp. 21622167, 2010.

[12] X. F. Wang and G. Chen, "Synchronization in small-world dynamical networks," International Journal of Bifurcation and Chaos, vol. 12, pp. 187-192, 2002.

[13] X. F. Wang and G. Chen, "Synchronization in scale-free dynamical networks: robustness and fragility," IEEE Transactions on Circuits and Systems. I, vol. 49, no. 1, pp. 54-62, 2002.

[14] C. G. Li and G. Chen, "Synchronization in general complex dynamical networks with coupling delays," Physica A, vol. 343, no. 1-4, pp. 263-278, 2004.

[15] C. W. Wu and L. O. Chua, "Synchronization in an array of linearly coupled dynamical systems," IEEE Transactions on Circuits and Systems. I, vol. 42, no. 8, pp. 430-447, 1995.

[16] C. W. Wu, "Synchronization in arrays of coupled nonlinear systems with delay and nonreciprocal timevarying coupling," IEEE Transactions on Circuits and Systems II, vol. 52, pp. 282-286, 2005.

[17] J. Cao, P. Li, and W. Wang, "Global synchronization in arrays of delayed neural networks with constant and delayed coupling," Physical Letters A, vol. 353, pp. 318-325, 2006.

[18] J. Lu and J. Cao, "Adaptive synchronization in tree-like dynamical networks," Nonlinear Analysis. Real World Applications, vol. 8, no. 4, pp. 1252-1260, 2007.

[19] S. H. Strogatz, "Exploding complex networks," Nature, vol. 410, pp. 268-276, 2001.

[20] M. Fiedler, "Algebraic connectivity of graphs," Czechoslovak Mathematical Journal, vol. 23, no. 98, pp. 298-305, 1973.

[21] F. R. K. Chung, Spectral Graph Theory, Conference Board of the Mathematical Sciences, American Mathematical Society, Providence, RI, USA, 1997.
[22] R. Merris, "Laplacian matrices of graphs: a survey," Linear Algebra and its Applications, vol. 197-198, pp. 143-176, 1994.

[23] B. Mohar, "The Laplacian spectrum of graphs," in Graph Theory, Combinatorics, and Applications, pp. 871-898, Wiley, New York, NY, USA, 1991. 


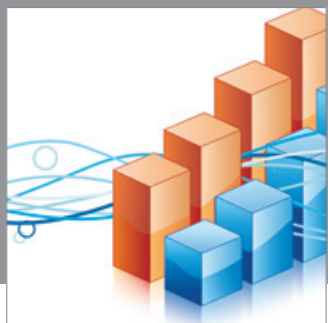

Advances in

Operations Research

mansans

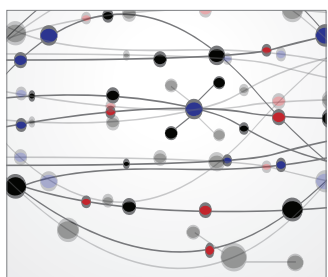

The Scientific World Journal
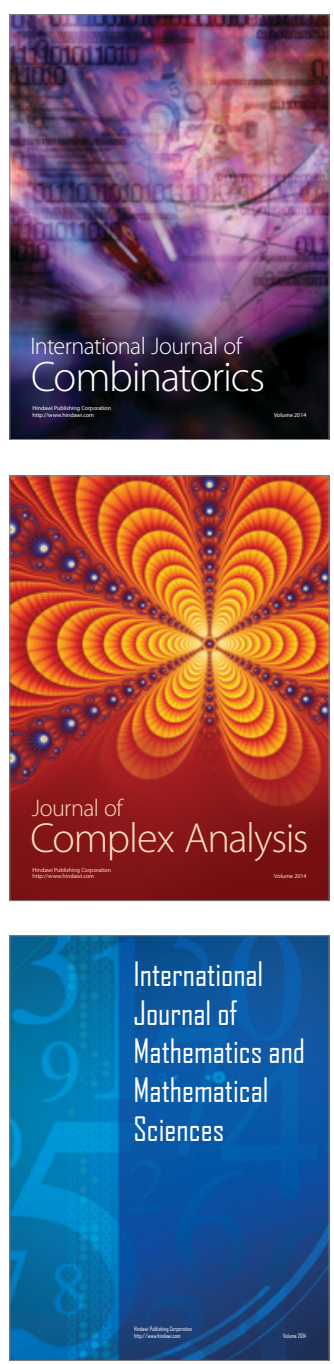
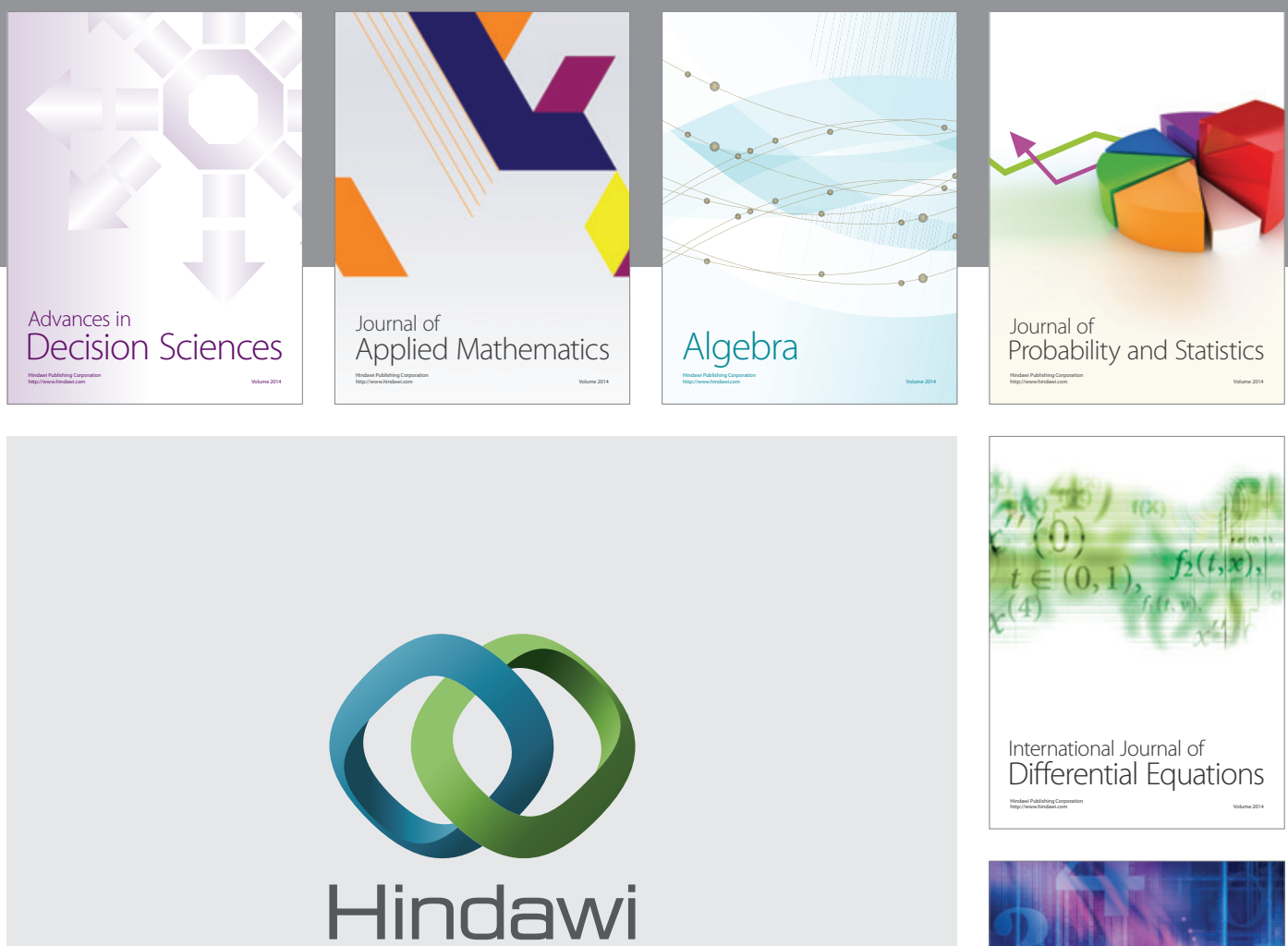

Submit your manuscripts at http://www.hindawi.com
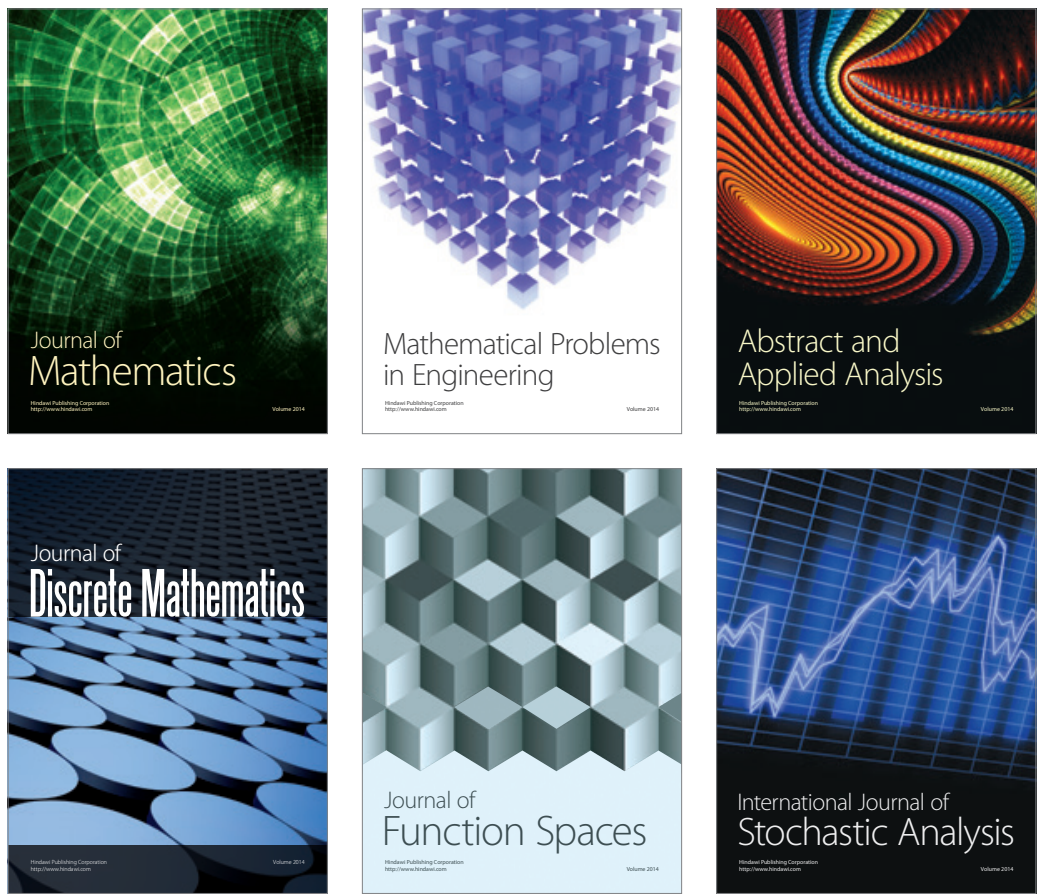

Journal of

Function Spaces

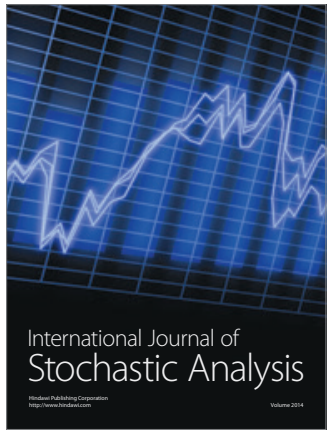

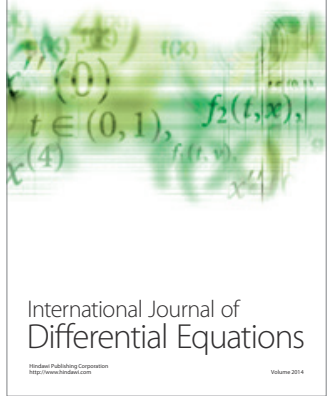
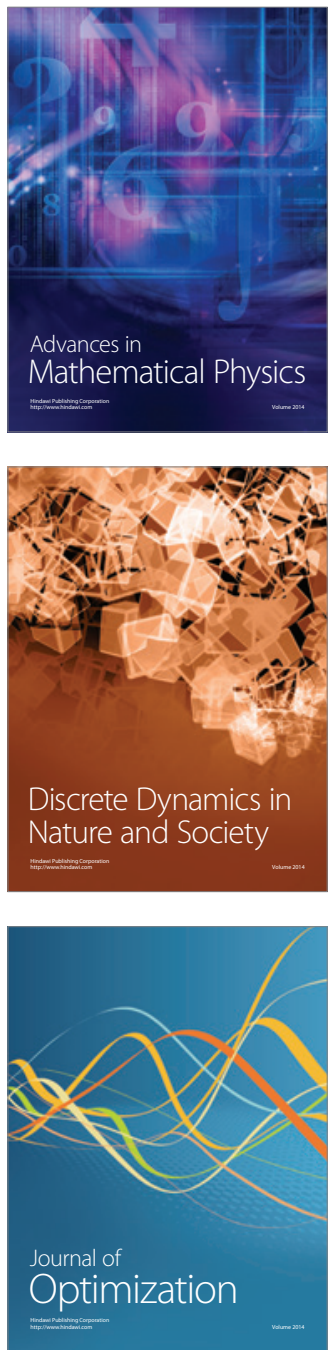